\title{
Theory for Axial Rigidity of Structural Members Having Ovaloid or Square Perforations
}

\author{
By Martin Greenspan
}

\begin{abstract}
Formulas are derived for computing the over-all lengthening (or shortening) of a tension (or compression) member having a uniform gross cross section and a series of similar perforations of approximately ovaloid or approximately square shape uniformly distributed along the length.
\end{abstract}

\section{Introduction}

In a previous paper [1] ${ }^{1}$ the author demonstrated a method for computing the axial rigidity of a tension or compression member having a uniform gross cross section and a series of similar perforations of circular or elliptical shape uniformly distributed along the length. The axialrigidity factor $K$ is defined so that $K E A_{g}$ is the rigidity that should be used in place of $E A_{g}$ in the ordinary formula for computation of the extension of the member. Here $E$ is Young's modulus of elasticity, and $A_{\mathrm{g}}$ is the gross cross-sectional area. The present paper extends this method to other shapes of perforation, originally described in reference [2]. The boundary of the hole has the parametric equation

$$
x=p \cos \beta+r \cos 3 \beta, y=q \sin \beta-r \sin 3 \beta .
$$

Equation 1 represents a closed curve having symmetry about the $x$-axis and about the $y$-axis, and which, for appropriate values of $p, q$, and $r$, does not cross itself. By adjustment of the values of $p, q$, and $r$, a variety of curves is obtained, including a good approximation to an ovaloid (a square with a semicircle erected on each of two opposite sides) and a good approximation to a square with rounded corners, as well as exact

1 Numbers in brackets indicate literature references at the end of this paper. ellipses $(r=0)$ of any eccentricity. The approximate ovaloid obtained by taking

$$
p=2.063, q=1.108, r=-0.079,
$$

is shown compared to the actual ovaloid in figure 1. The approximate square obtained by taking

$$
p=q=1, r=-0.14
$$

is shown in figure 2. The sides of the square are parallel to the axes of coordinates. By taking

$$
p=q=1, r=0.14,
$$

\section{Contents}

I. Introduction

II. Displacements _._. 158

1. Displacement-function method ....... 158

2. Mushelisvili's method _..._._._._. 159

III. Axial rigidity _._. 160

1. General _....... 160

2. Ovaloid; load parallel to long axis_..... 160

3. Ovaloid; load parallel to short axis _ . . . 161

4. Square with rounded corners _......... 161

IV. Summary _.................. 161

V. References _........ 161 
the same square, but with the diagonals parallel to the axes of coordinates, is obtained. The radius of curvature at the midpoint of the fillet is about 0.086 times the length of the side of the square.

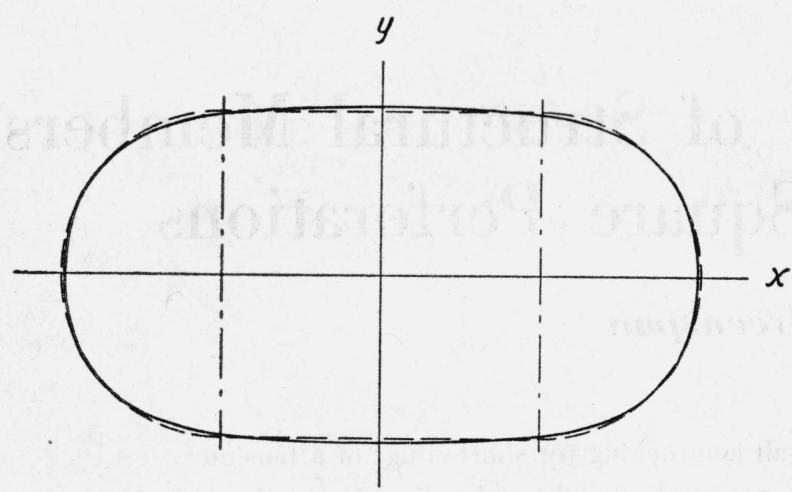

FIgUre 1.-Actual and approximate ovaloids.

The dashed line represents the actual ovaloid and the full line the approx imate ovaloid of equations 1 and 2

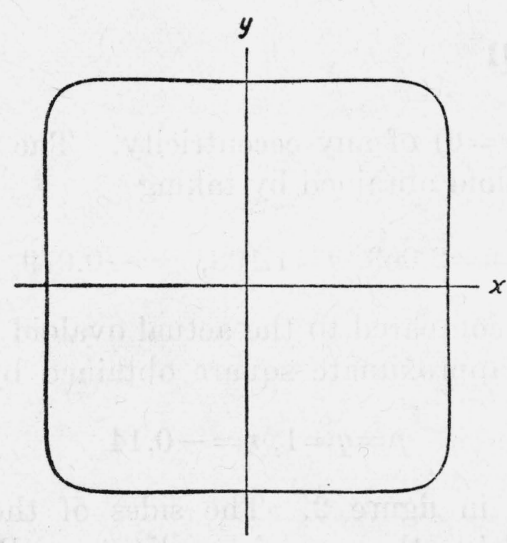

FIGURE 2.-The approximate square of equations 1 and 3.
It is shown in $[1, \text { eq } 2]^{2}$ that for a member having perforations spaced $2 l$ apart along the $x$-axis (parallel to the load), the axial rigidity factor $K$ is given by

$$
\frac{1}{K}=1+\frac{E}{P l} \int u_{p} d y d z,
$$

in which $u_{p}$ is the $x$-component of the displacement of the boundary of the hole, and the surface integral is extended over half the boundary of the perforation. The values of the load $P$ and the displacement $u_{p}$ may be assumed, for sufficiently large values of $l$ and of $A_{g}$ compared to the dimensions of the perforation, to be the same as in an infinite plate under uniform stress $S_{x}$ disturbed by a single perforation.

For a member having circular holes, and of gross cross-sectional area $A_{g}$ and net cross-sectional area $A_{n}$,

$$
P=S_{x} A_{g} C(n)=S_{x} A_{g}\left(1-\frac{1}{2 n^{2}}-\frac{1}{2 n^{4}}\right)
$$

where $S_{x}$ is the mean stress on the gross crosssectional area and $n=\mathrm{A}_{g} /\left(A_{g}-A_{n}\right)$. Equation 6 is derived in [1]. $C(n)$ may be considered to be a correction factor arising from the finite area of the member. It has been shown $[3]^{3}$ that $C(n)$ is nearly independent of eccentricity in case of an elliptical hole, and since it is nearly unity in any practical case, eq 6 may be used without serious error for a hole of any shape.

The displacement $u_{p}$ may be calculated from the stress function given in [2] or by the method of Mushelisvili.

2 This notation is used throughout for the numbered equations in the references.

${ }^{3}$ See p. 545 of reference [3].

\section{Displacements}

\section{Displacement-function method}

This method ${ }^{4}$ presupposes a knowledge of the Airy stress-function, $\phi$. The form of $\phi$ is exhibited in [2] in terms of curvilinear coördinates $(\alpha, \beta)$ such that eq 1 of the boundary of the hole reduces to $\alpha=\alpha_{0}$. The parameters $a, b$, and $c$ of [2], which, together with $\alpha_{0}$, define the shape of the hole, are related to the $p, q$, and $r$ of eq 1 by

$$
e^{\alpha_{0}}=\frac{p+q}{2}, a b e^{-\alpha_{0}}=\frac{p-q}{2}, a c^{3} e^{-3 \alpha_{0}}=r .
$$

\footnotetext{
4 See p. 130 of [3].
}

The function $\psi$ defined, in the absence of body forces, by

$$
\frac{\partial^{2} \psi}{\partial x \partial y}=\frac{\partial^{2} \phi}{\partial x^{2}}+\frac{\partial^{2} \phi}{\partial y^{2}}=\nabla^{2} \phi
$$

with $\psi$ adjusted by means of the arbitrary functions of integration (of the form $f_{1}(x)+f_{2}(y)$ ) so that $\nabla^{2} \psi=0$, is called the displacement function. The $x$-component of the displacement is given by

$$
u=\frac{1}{E} \frac{\partial \psi}{\partial y}-(1+\nu) \frac{\partial \phi}{\partial x}
$$


where $\mu$ is Poisson's ratio. In the present case the only values of $u$ required are $u_{p}$, those on the boundary of the perforation. On this (free) boundary, $\partial \phi / \partial x$ is constant, and hence zero, since $\phi$ is even in $x$ and in $y$. Hence from eq 9 ,

$$
E u_{p}=\frac{\partial \psi}{\partial y}\left(\alpha=\alpha_{0}\right)
$$

According to [2, eq 18],

$$
\begin{aligned}
\phi= & C_{1} \phi_{1}+C_{2} \phi_{2}+C_{3} \phi_{3}+C_{4} \phi_{4}+ \\
& C_{5} \phi_{5}+C_{6} \phi_{6}+C_{7} \phi_{7}+C_{8} \phi_{8} .
\end{aligned}
$$

Of these terms, all but those in $\phi_{1}, \phi_{2}$, and $\phi_{6}$ are harmonic and need not be considered for purposes of eq 8. Further, $C_{6}=0$ for no shear, as shown by [2, eq 23]. Thus only the terms in $\phi_{1}$ and $\phi_{2}$ are required. From paragraph 5 of [2] $\phi_{1}$ and $\phi_{2}$ are, omitting harmonic terms, which disappear for operation $\nabla^{2}$,

$$
\phi_{1}=4 y e^{\alpha} \sin \beta-4 a b y e^{-\alpha} \sin \beta-x^{2}-y^{2},
$$

and

$$
\phi_{2}=2 y e^{-\alpha} \sin \beta,
$$

so that

$$
\nabla^{2} \phi_{1}=8 \frac{\partial}{\partial y}\left(e^{\alpha} \sin \beta-a b e^{-\alpha} \sin \beta\right)-4,
$$

and

$$
\nabla^{2} \phi_{2}=-4 \frac{\partial}{\partial y} e^{-\alpha} \sin \beta .
$$

Equation 8 becomes

$$
\begin{aligned}
\frac{\partial^{2} \psi}{\partial x \partial y} & =C_{1}\left[8 \frac{\partial}{\partial y}\left(e^{\alpha} \sin \beta-a b e^{-\alpha} \sin \beta\right)-4\right] \\
& -4 C_{2} \frac{\partial}{\partial y} e^{-\alpha} \sin \beta,
\end{aligned}
$$

from which

$$
\begin{aligned}
\frac{\partial \psi}{\partial x} & =C_{1}\left[8\left(e^{\alpha} \sin \beta-a b e^{-\alpha} \sin \beta\right)-4 y\right] \\
& -4 C_{2} e^{-\alpha} \sin \beta+D x+E,
\end{aligned}
$$

in which $D x+E$ is the most general function of integration of the form $f_{1}(x)$ which preserves the harmonic character of $\partial \psi / \partial x$. Because $\psi$ is harmonic by definition, $\partial \psi / \partial y$ as well as $\partial \psi / \partial x$ is harmonic, and application of the CauchyRiemann test to the expression

$$
\frac{\partial \psi}{\partial y}+i \frac{\partial \psi}{\partial x}
$$

shows it to be a function of the complex variable $x+i y$. Thus $\partial \psi / \partial y$ is the real part of the function of which $\partial \psi / \partial x$ is the imaginary part, so that from eq 11

$$
\begin{aligned}
\frac{\partial \psi}{\partial y} & =C_{1}\left(8 e^{\alpha} \cos \beta+8 a b e^{-\alpha} \cos \beta-4 x\right) \\
& +4 C_{2} e^{-\alpha} \cos \beta-D y+E^{\prime} .
\end{aligned}
$$

The constants $D$ and $E^{1}$ are obtained by substituting for $\partial \psi / \partial y$ in eq 9 , which gives

$$
\begin{aligned}
E u & =C_{1}\left(8 e^{\alpha} \cos \beta+8 a b e^{-\alpha} \cos \beta-4 x\right) \\
& +4 C_{2} e^{-\alpha} \cos \beta-D y+E^{\prime}-(1+\nu) \frac{\partial \phi}{\partial x} .
\end{aligned}
$$

For $x=0(\beta= \pm \pi / 2), u=0$ for all $y$, and $\partial \phi / \partial x=0$ by symmetry. Therefore, $D=E^{\prime}=0$, and

$$
\begin{aligned}
\frac{\partial \psi}{\partial y}= & C_{1}\left(8 e^{\alpha} \cos \beta+8 a b e^{-\alpha} \cos \beta-4 x\right) \\
& +4 C_{2} e^{-\alpha} \cos \beta .
\end{aligned}
$$

Substitution of eq 12 , with $\mathrm{C}_{1}$ and $\mathrm{C}_{2}$ replaced by their values from [2, eq 23] into eq 10 gives

$$
\frac{E u_{p}}{S_{x}}=-x_{0}+\frac{2 e^{\alpha_{0}} \cos \beta}{1-a c^{3} e^{-4 \alpha_{0}}}\left(2-a c^{3} e^{-4 \alpha_{0}}-a^{2} b c^{3} e^{-6 \alpha_{0}}\right),
$$

where $x_{0}$ is the value of $x$ on the boundary $\alpha=\alpha_{0}$, which, together with eq 7 , gives

$$
\frac{E u_{p}}{S_{x}}=-x_{0}+2 \frac{(p+q)^{2}-2 p r}{p+q-2 r} \cos \beta .
$$

\section{Mushelisvili's method}

This method, which is treated in detail by Sokolnikoff [4], expresses the stresses, displacements, and the boundary conditions in terms of two analytic functions, $\phi$ and $\psi$ (not the same as those of the preceding section), of the complex variable $z=x+i y$. The functions $\phi$ and $\psi$, which satisfy the problem of [2], have been given by Morkovin [5], who uses the notation

and

$$
s=(p+q) / 2, t=(p-q) / 2, r=r,
$$

$$
\zeta=e^{\alpha-\alpha_{0}}+i \beta
$$

so that the function

$$
z=\omega(\zeta)=s \zeta+t / \zeta+r / \zeta^{3}
$$

maps the boundary $\alpha=\alpha_{0}$ onto the unit circle $|\zeta|=1$. The value of $\zeta$ on the unit circle is denoted by $\sigma$; thus $\bar{\sigma}$, the conjugate of $\sigma$, is $1 / \sigma$. The notation

$$
\phi(z)=\phi[\omega(\zeta)]=\phi(\zeta)
$$

is used, and similarly for $\psi$. 
The functions $\phi$ and $\psi$ as found by Morkovin are

$$
\begin{gathered}
\phi(\zeta)=s B \zeta+\frac{a_{1}}{\zeta}-\frac{B r}{\zeta^{3}} \\
\psi(\zeta)=-\left(\frac{s}{\zeta}+t \zeta+r \zeta^{3}\right)\left(\frac{s B \zeta^{4}-a_{1} \zeta^{2}+3 r B}{s \zeta^{4}-t \zeta^{2}-3 r}\right)-\frac{s B}{\zeta} \\
+s\left(B^{\prime}+i T_{x y}\right) \zeta-a_{1} \frac{r}{s} \zeta+B r \zeta^{3}+B i\left(1+\frac{r}{s}\right) \zeta
\end{gathered}
$$

where

and

$$
a_{1}=B t \frac{r+s}{r-s}+\frac{B^{\prime} s^{2}}{r-s}+\frac{i T_{x y} s^{2}}{r+s},
$$

$$
4 B=S_{x}+S_{y}, 2 B^{\prime}=S_{y}-S_{x} .
$$

In the present case, $S_{\nu}=T_{x y}=0$, giving

$$
\begin{gathered}
\phi(\zeta)=\frac{S_{x}}{4}\left(s \zeta+\frac{a}{\zeta}-\frac{r}{\zeta^{3}}\right) \\
\psi(\zeta)=\frac{S_{x}}{4}\left[-\left(\frac{s}{\zeta}+t \zeta+r \zeta^{3}\right)\left(\frac{s \zeta^{4}-a \zeta^{2}+3 r}{s \zeta^{4}-t \zeta^{2}-3 r}\right)\right. \\
\left.-\frac{s}{\zeta}-2 s \zeta-\frac{a r}{s} \zeta+r \zeta^{3}+t\left(1+\frac{r}{s}\right) \zeta\right]
\end{gathered}
$$

where

$$
a=\frac{4 a_{1}}{S_{x}}=\frac{t(r+s)-2 s^{2}}{r-s} .
$$

\section{Axial Rigidity}

\section{General}

Substitution of $P$ from eq 6 and $u_{p}$ from eq 13 into eq 5 gives

$$
\begin{aligned}
\frac{1}{K}=1+\frac{t}{A_{g} l C(n)} & {\left[-\int x d y\right.} \\
+ & \left.2 \frac{(p+q)^{2}-2 p r}{p+q-2 r} \int \cos \beta d y\right],
\end{aligned}
$$

where $t$, the thickness of the plate in which the perforation occurs, is $\int d z$. The two line integrals in eq 21 , which are to be taken along half the boundary $\alpha=\alpha_{0}$, are obtained from eq 1 , as

$$
\int x d y=\frac{1}{2} A_{0}=\frac{\pi}{2}\left(p q-3 r^{2}\right), \int \cos \beta d y=\frac{\pi q}{2},
$$

where $A_{0}$ is the area of the perforation.

Equation 21 becomes

$$
\overline{1}=1+\frac{V_{0}}{V_{g} C(n)} \frac{q(p+q)(p+2 q)}{(p+q-2 r)\left(p q-3 r^{2}\right)},
$$

The rectangular components, $u$ and $v$, of the displacement are expressed in terms of the functions $\phi$ and $\psi$ by means of the relation

$$
E(u+i v)=(3-\nu) \phi(\zeta)-(1+\nu)\left[\frac{z \bar{\phi}^{\prime}(\bar{\zeta})}{\overline{\omega^{\prime}}(\bar{\zeta})}+\bar{\psi}(\bar{\zeta})\right],
$$

so that on the boundary $\zeta=\sigma$,

$$
\begin{aligned}
E\left(u_{p}+i v_{p}\right) & =(3-\nu) \phi(\sigma) \\
& -(1+\nu)\left[\frac{\overline{z \phi^{\prime}}(\bar{\sigma})}{\overline{\omega^{\prime}}(\bar{\sigma})}+\bar{\psi}(\bar{\sigma})\right] .
\end{aligned}
$$

Substitution of the functions $\omega, \phi$, and $\psi$ from eq 16,17 , and 18 in eq 19 yields, after considerable reduction,

$$
\frac{E}{S_{x}}\left(u_{p}+i v_{p}\right)=-z_{0}+2 s \sigma+\frac{2 s^{2}-2 r t}{s-r} \frac{1}{\sigma},
$$

where $z_{0}$ is on $\alpha=\alpha_{0}$, and therefore,

$$
\frac{E u_{p}}{S_{x}}=-x_{0}+\frac{4 s^{2}-2 s r-2 r t}{s-r} \cos \beta,
$$

since $\sigma=e^{1 \beta}$ from eq 15 .

In terms of the original constants (eq 14), eq 20 becomes,

$$
\frac{E u_{p}}{S_{x}}=-x_{0}+2 \frac{(p+q)^{2}-2 p r}{p+q-2 r} \cos \beta,
$$

which is the same as eq 13 .

where $V_{0}=A_{0} t$ is the volume of the perforation and $V_{g}=2 A_{g} l$ is the gross volume of one bay of the member.

For an ellipse, $p=a, q=l, r=0$, where $a$ and $b$ are the semiaxes ( $a$ parallel to the load) and eq 22 reduces to

$$
\frac{1}{K}=1+\frac{1+\frac{2 b}{a} V_{0}}{C(n)} \bar{V}_{g}
$$

which is the same as [1, eq 48].

It remains to compute the coefficient of $V_{0} / V_{g}$ in eq 22 for the various cases of interest.

\section{Ovaloid; load parallel to long axis}

Here $p=2.063, q=1.108, r=-0.079$ (eq 2). Equation 22 becomes

$$
\frac{1}{K}=1+\frac{2.048}{C(n)} \frac{V_{0}}{V_{g}}
$$

In practical numerical cases, eq 23 gives substantially the same results as [1, eq 13], derived 
by a more approximate method. However [1, eq 13] may be used for an ovaloid of which the rectangular base is not square whereas eq 23 applies only to the shape shown in figure 1.

\section{Ovaloid; load parallel to short axis}

In this case, $p=1.108, q=2.063, r=0.079$. Equal 23 becomes

$$
\frac{1}{K}=1+\frac{4.968 V_{0}}{C(n) V_{g}}
$$

This case and the following cases are not covered by previous work.

\section{Square with Rounded Corners}

For the approximate square of eq 3 and figure $2, p=q=1, r=-0.14$. Eq. 22 becomes

$$
\frac{1}{K}=1+\frac{2.989}{C(n)} \frac{V_{0}}{V_{g}}
$$

for load parallel to the side of the square.

For load parallel to the diagonal of the square (eq 4) $p=g=1, r=0.14$, and eq 22 becomes

$$
\frac{1}{K}=1+\frac{3.596}{C(n)} \frac{V_{0}}{V_{g}} .
$$

\section{Summary}

Approximate formulas have been developed for the computation of the axial rigidity of a long tension or compression member containing a plate of constant thickness uniformly perforated with a series of similar holes of various shapes. The axial rigidity factor $K$ is defined so that $K E A_{g}$ is the rigidity that should be used in place of $E A_{g}$ in the ordinary formula for computation of the extension of the member.

In many cases of interest the axial rigidity factor $K$ is given by an equation of the form

$$
\frac{1}{K}=1+\frac{f}{C(n)} \frac{V_{0}}{V_{g}}
$$

where

$n=A_{g} /\left(A_{g}-A_{n}\right), A_{g}$ being the gross and $A_{n}$ the net cross-sectional area of the member
$C(n)=1-\frac{1}{2 n^{2}}-\frac{1}{2 n^{4}}$

$V_{o}=$ the volume of the perforation

$V_{g}=$ the gross volume of one bay of the member $f=a$ constant depending on the shape of the perforation and the direction of the applied load.

\begin{tabular}{|c|c|c|}
\hline Perforation & Load parallel to- & $f$ \\
\hline Ellipse of semiaxes $a$ and $b$. & Major axis, $a_{-.}$ & $1+(2 b / a)$ \\
\hline Do & Minor axis, $b$ & $1+(2 a / b)$ \\
\hline Ovaloid (fig. 1) & Long axis. & 2. 048 \\
\hline Do & Short axis ........ & 4. 968 \\
\hline Square (fig. 2) & Side & 2. 989 \\
\hline Do. & Diagonal & 3. 596 \\
\hline
\end{tabular}

Values of the constant $f$ for various cases are given in table 1 .

TABLE 1.-Values of $f$ in equation 27

\section{References}

[1] Martin Greenspan, Axial rigidity of perforated structural members, J. Research NBS 31, 305 (1943) RP1568.

[2] Martin Greenspan, Effect of a small hole on the stresses in a uniformly loaded plate, Quart. Applied Math. II, 60 (1944).

[3] E. G. Coker and L. N. G. Filon, A treatise on photoelasticity (Cambridge, 1931).
[4] I. S. Sokolnikoff, Mathematical theory of elasticity, (McGraw-Hill Book Co., Inc., New York, N. Y. 1946).

[5] Vladimir Morkovin, Effect of a small hole on the stresses in a uniformly loaded plate, Quart. Applied Math. II, 350 (1945).

Washington, May 21, 1946. 